Reprod. Nutr. Dévelop., 1986, 26 (5 B), 1151-1162.

\title{
Rôle du système nerveux autonome dans la régulation du transit, de l'absorption et du stockage des nutriments
}

N. MEI

Laboratoire de Neurobiologie - Interoception, C.N.R.S.

31. Chemin Joseph Aiguier, 13402 Marseille Cedex 9, France.

Summary. Role of the autonomic nervous system in the regulation of gastrointestinal motility, absorption and storage of nutriments.

The possible role of the autonomic nervous system (ANS) in nutrition must be reevaluated in view of recent experimental data.

The ANS plays a major part in both initiating and maintaining peristalsis and in coordinating gastrointestinal motility.

Intestinal absorption involves extra-epithelial mechanisms such as motility and vastomotricity of the digestive tract. In addition, the ANS (sympathetic fibres) might act directly on enterocytes, or indirectly via the intestinal plexuses through a glucosedependent mechanism.

The control of exocrine and endocrine secretions depends partly on the ANS. In particular the mucous mechanoreceptors, chemoreceptors and thermoreceptors located in the intestinal area supply the sensory information needed in that kind of regulation.

The efferent fibres of the ANS intervene in the control of storage of carbohydrates in the liver and of lipids in brown adipose tissue. On the other hand, gastrointestinal afferents might be implicated in this mechanism through the hypothalamic ventro-median nucleus.

Finally, these data are consistent with a modern conception suggesting that the ANS is largely involved in the regulation of visceral motility, homeostasis and alimentary behaviour.

\section{Introduction.}

Tous les ouvrages consacrés à la nutrition mettent en exergue la multiplicité et la complexité des mécanismes qui assurent les fonctions de nutrition chez les Mammifères. Cependant, l'aspect biochimique de la nutrition y est davantage développé que les autres aspects qui intéressent les régulations humorales et surtout nerveuses. Cet état de fait s'explique avant tout par le peu de données disponibles sur le système nerveux autonome dont les caractéristiques ont constitué autant d'obstacles à son étude. L'avènement des technologies modernes, mais également le regain d'intérêt des chercheurs pour le domaine viscéral, ont apporté 
un éclairage nouveau sur cette question à tel point qu'aujourd'hui la tendance est de considérer que le système nerveux autonome en général, et le système nerveux digestif en particulier, jouent un rôle important dans la régulation des mécanismes qui assurent le transit, l'absorption et le stockage des aliments.

Cet exposé ne constitue pas une étude exhaustive, mais une tentative pour rassembler quelques-uns des faits les plus significatifs démontrant ou suggérant le rôle du système nerveux dans la nutrition, ce qui, à notre connaissance, n'avait pas encore été réalisé. II comprend, de plus, un rappel de nos connaissances actuelles portant sur les propriétés morphologiques et fonctionnelles du système nerveux autonome. L'incorporation de ces données se justifie par une double raison : $\left.1^{\circ}\right)$ nombre d'idées considérées naguère comme classiques ont dû être sérieusement révisées a la suite des travaux récents $; 2^{\circ}$ ) ces considérations nouvelles ont été souvent à l'origine de recherches dans lesquelles le rôle du système nerveux autonome dans la nutrition a été réexaminé.

\section{Données actuelles sur l'innervation du système nerveux gastro-intestinal}

Le système nerveux gastro-intestinal, comme d'ailleurs l'ensemble du système nerveux végétatif, a été longtemps très mal connu, en raison des difficultés de toute nature que son étude présentait. Certains obstacles sont d'ordre morphologique ; rappelons, en particulier, qu'une grande majorité des fibres végétatives sont de petite taille (fibres myélinisées de petit diamètre et surtout fibres amyéliniques). Or ce type de fibres se prête mal à l'observation histologique aussi bien qu'à l'investigation électrophysiologique fine. D'autres difficultés, qui sont d'ordre fonctionnel, tiennent notamment à la concomitance des mécanismes biochimiques, humoraux et nerveux qui rend particulièrement malaisée l'étude de la nutrition chez l'animal entier. Dans ce chapitre, nous analyserons successivement les données qui ont trait aux deux composantes du système nerveux digestif : le système intrinsèque et le système extrinsèque.

\section{1) Le système nerveux intrinsèque.}

Le système nerveux intrinsèque avec ses deux plexus, le plexus myentérique et le plexus sous-muqueux, constitue en fait un véritable centre, que certains auteurs qualifient même de " cerveau périphérique ". Les études ultrastructurales et histochimiques modernes ont bien démontré la richesse et la diversité de ce système. Chez l'Homme par exemple, on a estimé le nombre de neurones, uniquement dans l'intestin, à quelques 100 millions de cellules (Furness et Costa, 1980) ! Au plan fonctionnel, ces neurones appartiennent à divers types suivant qu'ils sont moteurs, sensitifs ou qu'ils jouent le rôle d'interneurones. Selon la nature du médiateur qu'ils synthétisent, les neurones intramuraux se différencient en plusieurs sous-groupes (neurones cholinergiques, adrénergiques, peptidergiques, sérotoninergiques ou purinergiques), les uns étant excitateurs, les autres inhibiteurs (Gershon, 1981). Quoi qu'il en soit, ces neurones contractent entre eux des relations complexes qui confèrent aux deux plexus un haut degré d'automa- 
tisme puisqu'à eux seuls, ils sont capables d'induire et de maintenir, au moins en partie, l'activité gastro-intestinale.

\section{2) Le système nerveux extrinsèque.}

Les travaux effectués au cours des deux dernières décennies ont mis en évidence la complexité remarquable du système nerveux autonome. Les trois points que nous développerons ici montrent bien cette complexité et illustrent, de plus, l'évolution récente des idées sur le système nerveux autonome. II s'agit de l'abondance des fibres sensitives, du fonctionnement des intérocepteurs et de l'organisation fonctionnelle des systèmes sympathique et parasympathique.

A) Abondance des fibres sensitives par rapport aux fibres motrices. - La proportion des fibres sensitives par rapport aux fibres motrices est plus grande qu'on ne le pensait. On trouve, par exemple, $80 \%$ de fibres sensitives pour seulement $20 \%$ de fibres efférentes dans le nerf vague (Agostoni et al., 1957; Mei et al., 1971). Ce fait s'explique par la présence de nombreuses fibres amyéliniques (80\% pour le nerf vague par exemple) dont le nombre avait été largement sousestimé, de sorte qu'on peut affirmer aujourd'hui que les principaux nerfs du tube digestif (vagues et splanchniques) sont principalement sensitifs, et non moteurs comme on le pensait naguère.

B) Fonctionnement des intérorécepteurs digestifs. - Parce que leur structure s'apparentait à celles des terminaisons libres de la peau, on a longtemps pensé que les intérocepteurs devaient intervenir essentiellement dans la douleur. En fait,

\section{TABLE 1}

Caractéristiques des récepteurs sensitifs décrits dans le tractus gastro-intestinal (voir en particulier, Paintal, 1973 ; Leek, 1977 et Mei, 1985).

\begin{tabular}{|c|c|c|c|}
\hline Type & Situation & $\begin{array}{l}\text { Trajet de } \\
\text { leur fibre }\end{array}$ & Stimulus \\
\hline Mécanorécepteurs & $\begin{array}{l}\text { Muqueuse, musculeuse, } \\
\text { séreuse }\end{array}$ & $\begin{array}{l}\text { N. vagues } \\
\text { N. splanchniques }\end{array}$ & $\begin{array}{l}\text { - Distension } \\
\text { - Contraction } \\
\text { - Déplacement } \\
\text { - Contact aliments }\end{array}$ \\
\hline Chémorécepteurs & Muqueuse & $\begin{array}{l}\text { N. vagues } \\
\text { N. splanchniques }\end{array}$ & $\begin{array}{l}\text { - Acides, bases, } \mathrm{pH} \\
\text { - Glucides } \\
\text { - Acides aminés } \\
\text { - Lipides }\end{array}$ \\
\hline Thermorécepteurs & Muqueuse, séreuse & $\begin{array}{l}\text { N. vagues } \\
\mathrm{N} \text {. splanchniques }\end{array}$ & $\begin{array}{l}\text { - Chaud } \\
\text { - Froid } \\
\text { - Chaud et froid }\end{array}$ \\
\hline Récepteurs polymodaux & Muqueuse & N. vagues & $\begin{array}{l}\text { - La plupart des stimu- } \\
\text { lus ci-dessus } \\
\text { - Osmolarité }\end{array}$ \\
\hline
\end{tabular}


I'emploi des techniques microphysiologiques ( ${ }^{1}$ ) a complètement infirmé ce point de vue. La plupart des récepteurs viscéraux sont actifs dans les conditions physiologiques normales. Ainsi, dans le tractus gastro-intestinal, divers types de mécanorécepteurs, de chémorécepteurs et de thermorécepteurs ont été décrits (Mei, 1983, 1985 et tabl. 1). II s'agit de récepteurs dits spécifiques car ils ne répondent qu'à une modalité sensorielle principale (mécanique, chimique ou thermique). Cette richesse est encore accrue du fait qu'à l'intérieur de chacun de ces trois groupes coexistent plusieurs variétés de récepteurs (par exemple pour les chémorécepteurs: les glucorécepteurs, les récepteurs alcalino ou acido-sensibles, les récepteurs aux acides aminés, les récepteurs aux acides gras). Ajoutons qu'il existe aussi des récepteurs non spécifiques activés par plusieurs modalités sensorielles - si ce n'est par la totalité. Ces terminaisons, qui ont été qualifiées de polymodales, semblent comprendre plusieurs types différents, parmi lesquels les récepteurs osmosensibles occupent une place privilégiée.

Les récepteurs ont été rencontrés dans toutes les couches du tractus gastrointestinal : muqueuse, musculeuse et séreuse, mais les plus nombreux ont une localisation muqueuse. Finalement, cet équipement complexe confère une richesse et une diversité remarquables à l'information sensorielle issue du tractus gastro-intestinal.

C) Organisation fonctionnelle des systèmes sympathique et parasympathique. - La classification entre fibres sympathiques adrénergiques exerçant un effet inhibiteur et fibres parasympathiques cholinergiques exerçant un effet excitateur a dû être reconsidérée. Par exemple, certaines fibres parasympathiques activent des neurones intramuraux inhibiteurs dont le médiateur n'est ni l'acétylcholine ni l'adrénaline mais I'ATP ou une substance voisine (Gonella, 1978). Autre fait qui vient compliquer le schéma classique, certaines fibres sympathiques agissent non pas sur le muscle lisse comme on le pensait, mais sur certains neurones intramuraux parasympathiques activateurs. Par sa remarquable diversité d'action (effets inhibiteurs sur le muscle lisse et effets activateurs ou inhibiteurs sur les plexus), le système nerveux extrinsèque peut moduler d'une façon très souple les fonctions digestives.

\section{Rôle du système nerveux autonome}

Nous examinerons successivement le rôle du système nerveux autonome dans le transit gastro-intestinal, dans l'absorption intestinale, dans les sécrétions digestives exocrines et endocrines et dans le stockage des nutriments dans l'organisme.

\section{1) Le transit gastro-intestinal.}

La motricité digestive, qui dépend étroitement du système nerveux autonome, assure la propulsion des aliments tout au long du système digestif. Le

(1) Deux techniques sont principalement employées : la technique dite de la fibre isolée qui s'applique aux nerfs et la technique des microélectrodes qui s'applique surtout aux ganglions sensitifs. Cette dernière méthode a été utilisée dans notre laboratoire en raison de sa plus grande efficacité, notamment vis-à-vis des fibres amyéliniques. 
système nerveux intrinsèque joue le rôle essentiel dans le déclenchement du péristaltisme, mécanisme de base de la motricité digestive, alors que le système extrinsèque intervient principalement dans la modulation du péristaltisme et dans la coordination de la motricité digestive.

A) Déclenchement et modulation du péristaltisme. - Contrairement à ce qui se passe pour l'œsophage. les centres de commande du péristaltisme gastrique et intestinal se trouvent situés dans le viscère même (plexus myentérique et sousmuqueux). C'est la présence des aliments qui active les récepteurs mécanosensibles et chémosensibles de la muqueuse et qui met en jeu les circuits nerveux responsables de la contraction propulsive. La propagation du péristaltisme nécessite une coordination nerveuse complexe : activation en amont du bol et inhibition en aval, impliquant la participation de plusieurs types d'interneurones dont plusieurs sont maintenant bien identifiés (voir Wood, 1981). Le système nerveux extrinsèque n'a pas de rôle dans le déclenchement de ce mécanisme réflexe comme l'ont montré depuis longtemps les expériences réalisées sur l'intestin isolé. Mais, dans les conditions normales, il intervient pour ajuster l'activité motrice aux besoins périphériques. Ainsi, les fibres sympathiques et parasympathiques s'articulent sur les neurones intrinsèques pour renforcer ou diminuer la commande motrice. Les effets marqués obtenus par stimulation des nerfs vagues et splanchniques attestent de l'importance de ce système dans le contrôle de la motilité gastrointestinale. En revanche, le rôle des afférences gastro-intestinales a été plus difficile à montrer dans les conditions physiologiques. II faut dire que les effets moteurs, produits à partir de ces afférences, mobilisent les centres nerveux dont le fonctionnement n'est pas toujours compatible avec les exigences de l'expérimentation (anesthésie en particulier). De plus, l'implication des afférences était sous-estimée, pour ne pas dire ignorée, jusqu'à ce que l'on découvre la présence de nombreux récepteurs digestifs capables de fournir aux centres les informations chimiques et mécaniques nécessaires à la modulation fine de la commande motrice.

B) Coordination de la motilité gastro-intestinale. - Les mécanismes de nature nerveuse qui coordonnent l'activité motrice du tractus digestif sont particulièrement variés et nombreux du fait de la diversité morphologique et fonctionnelle de ce système. C'est ainsi que plusieurs réflexes activateurs ou inhibiteurs ont été décrits dans le tractus gastro-intestinal. Citons, par exemple, le réflexe activateur du côlon qui est déclenché par la réplétion de l'estomac (réflexe gastrocolique que l'on observe d'une façon très marquée chez le nourrisson), et le réflexe inhibiteur intestino-intestinal (Morin, 1935) qui se manifeste par une inhibition de la motricité de part et d'autre d'une zone de forte distension. Une mention spéciale doit être faite au réflexe inhibiteur entéro-gastrique en raison de l'intérêt qu'il a suscité au cours des dernières années. Après le réflexe de Thomas (1957), engendré par une distension du duodénum, on a mis en évidence d'autres réflexes analogues, déclenchés par les divers stimulus intestinaux connus, à savoir la présence de diverses substances : glucose, acides aminés, acides gras, les variations thermiques et les changements de la pression osmotique (Roman et Gonella, 1981 ; Kelly, 1981 ; Laplace et Cuber, 1984). Les connaissances actuelles, relatives à l'équipement sensitif du tube digestif, permettent désormais d'attri- 
buer l'origine de ces réflexes aux différents types de récepteurs décrits dans l'intestin (Mei, 1985). La multiplicité et la complémentarité des réflexes impliqués dans la régulation de la vidange gastrique soulignent l'importance fonctionnelle du complexe gastro-intestinal dans la digestion. Tout se passe, en somme, comme si le chyme était délivré sélectivement par l'estomac en fonction des capacités d'absorption optimales de l'intestin.

\section{2) L'absorption intestinale.}

L'absorption intestinale est, avant tout, un phénomène biochimique circonscrit à l'épithélium intestinal. Différents facteurs extra-épithéliaux peuvent, cependant, modifier l'absorption ; il s'agit de la motricité digestive, de la vasomotricité gastro-intestinale et des sécrétions digestives. Nous allons analyser le rôle du système nerveux dans ces deux types de mécanismes.

A) L'absorption proprement dite. - Le passage de l'eau, des électrolytes et des nutriments a été longtemps considéré comme un phénomène purement biochimique, lié aux propriétés membranaires et cytoplasmiques des entérocytes. II n'est donc pas étonnant que, dans un très grand nombre d'expériences, l'absorption ait été étudiée sur des segments isolés d'intestin (normaux ou éversés). Considérée uniquement sous cet angle, l'absorption paraissait complètement indépendante du système nerveux. En fait, les données récentes suggèrent que, dès ce stade, le système nerveux (extrinsèque et intrinsèque) pourrait intervenir. Sjövall et al. (1983a) ont montré que la stimulation des nerfs splanchniques provoquait, dans certaines conditions (perfusion intestinale avec une solution contenant du glucose), une augmentation du transport de $\mathrm{Na}^{+}$et des liquides sans changement de la motricité intestinale ni du débit sanguin. Ce phénomène, qui se produirait au cours de la digestion (Sjövall et al., 1983b), résulterait d'une action nerveuse s'exerçant directement sur l'épithélium intestinal (en effet, de fines formations adrénergiques ont été observées à proximité, Thomas et Templeton, 1981), ou indirectement, via les plexus intramuraux. Cette action se traduirait par une modification du transfert actif des nutriments, des électrolytes et de l'eau vers la circulation porte (absorption proprement dite) ou vers la lumière intestinale (sécrétion), phénomènes qui dépendent à la fois des mouvements ioniques membranaires et de l'activation enzymatique cellulaire (pompe à sodium et à potassium dont l'énergie est fournie par l'ATP). Si le rôle du système sympathique dans le contrôle de l'absorption semble ainsi démontré, on ne sait encore rien d'une intervention possible du système parasympathique. Toutefois, le nerf vague pourrait être concerné par ses afférences glucosensibles, dans la mesure où le glucose est associé à ce mécanisme (Sjövall et al., 1983b).

B) Les mécanismes extra-épithéliaux. - Les recherches modernes essayent de plus en plus de replacer l'intestin dans son contexte physiologique normal afin d'analyser les facteurs extra-épithéliaux susceptibles de modifier l'absorption. L'existence de tels facteurs était suggérée par le fait que l'absorption est en général plus importante dans les conditions in vivo que dans les conditions in vitro (Hajjar et Schedl, 1981). En dehors de la motricité digestive dont nous avons déjà 
parlé et des sécrétions digestives qui seront traitées plus loin, une place privilégiée doit être réservée à la vasomotricité abdominale.

La masse sanguine cœliaque et mésentérique est accrue au cours des repas (Vatner et al., 1970), ce qui favorise à la fois la sécrétion (surtout au niveau gastrique) et l'absorption (surtout au niveau intestinal).

Au niveau intestinal, l'absorption s'accompagne d'une vasodilatation qui intéresse surtout la région de la muqueuse et de la sous-muqueuse : c'est l'hyperémie postprandiale. Ce phénomène résulte de la mise en jeu de plusieurs mécanismes locaux dont le plus important semble être la régulation par le système nerveux entérique (Chou et al., 1972 ; Yu et al., 1975). Le rôle du système nerveux extrinsèque ainsi que la contribution respective du système sympathique et parasympathique ne sont pas clairement établis. Les résultats des expériences de stimulation des nerfs digestifs sont souvent difficiles à interpréter car les modifications vasomotrices observées peuvent n'être que la conséquence des phénomènes moteurs et sécrétoires ainsi engendrés. II est bien connu, par exemple, que la contraction de l'intestin s'accompagne d'une vasoconstriction localisée. Toutefois, la stimulation du système sympathique provoque une vasoconstriction marquée résultant d'un effet adrénergique (Furness et Costa, 1974) alors que la stimulation du système parasympathique entraîne des changements moins nets et plus variables (Tepperman et Jacobson, 1981). Quoi qu'il en soit, il est raisonnable de penser que le système nerveux extrinsèque intervient dans l'ajustement du débit circulatoire entre les différents segments de l'intestin et dans la redistribution des masses sanguines mésentérique et somatique. Dans la mesure où les récepteurs gastrointestinaux sont capables d'être activés par des substances, qui, comme le glucose, déclenchent des variations vasomotrices locales, les afférences splanchniques, et surtout vagales, pourraient être également impliquées dans la régulation de la circulation mésentérique.

\section{3) La régulation des sécrétions exocrines et endocrines liées à l'absorption.}

Les nutriments peuvent agir directement sur les glandes digestives, comme le fait le glucose sur les cellules pancréatiques. Mais généralement, ils interviennent alors même qu'ils sont encore dans la lumière gastrique ou intestinale. Dans ce dernier cas, leur action nécessite la participation d'un signal qui, à partir de la muqueuse digestive, va exercer son effet sur la glande concernée. Au cours de la période écoulée, on admettait que ce signal était essentiellement de nature hormonale, pour des raisons expérimentales : la section des nerfs digestifs affecte peu les sécrétions exo et endocrines, mais aussi et surtout par suite de l'engouement des chercheurs pour les hormones intestinales (Grossman, 1979). Les études récentes tendent non seulement à réhabiliter le rôle du système nerveux, mais à en privilégier le rôle, au moins pour certaines sécrétions. Les faits qui ont conduit à ce changement peuvent être ainsi résumés :

1) les expériences basées sur la stimulation électrique ont révélé les puissantes capacités sécrétoires du système nerveux extrinsèque ;

2) dans les conditions physiologiques normales, les techniques de dosage ne 
montrent pas toujours une augmentation significative de la libération des hormones intestinales, qui soit compatible avec un effet glandulaire ;

3) la rapidité de la réponse sécrétoire à l'arrivée dans le tractus gastro-intestinal des aliments, exclut l'intervention d'un mécanisme hormonal au moins au début. C'est le cas aussi bien des sécrétions exocrines (exemple des sécrétions pancréatiques, Singer et al., 1980) que des sécrétions endocrines (exemple de l'insuline, Mei et al., 1981);

4) il n'a pas encore pu être démontré que le phénomène d'adaptation des sécrétions à la composition des repas soit dû à une libération d'une hormone intestinale connue ;

5) on sait aujourd'hui que le tube digestif est doté d'un équipement sensoriel, riche et varié, susceptible de fournir toutes les informations utiles sur la composition du chyme. Les terminaisons nerveuses de tous types - en particulier les chémorécepteurs - peuvent donc représenter le point de départ de circuits réflexes impliqués dans les régulations sécrétoires.

En dépit de ces données, les mécanismes réflexes impliqués sont encore loin d'être connus d'une façon complète. Une exception doit cependant être faite : la régulation nerveuse de l'insulinémie à partir de l'intestin (fig. 1). Le point de

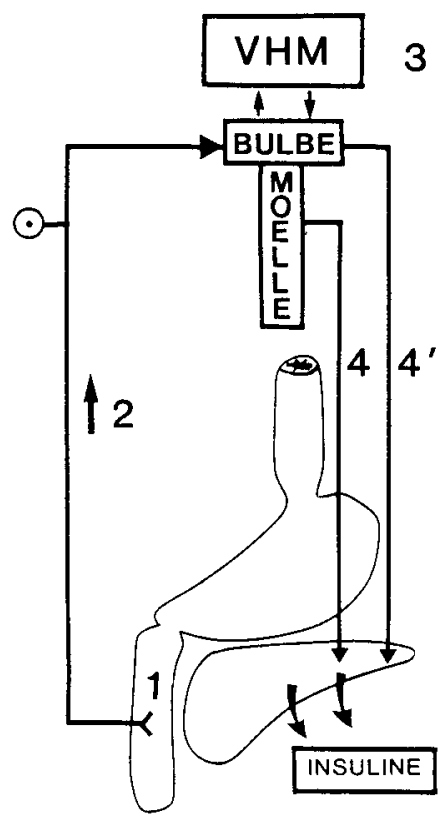

FIG.1. - Régulation nerveuse de l'insulinémie à point de départ intestinal.

1 : glucorécepteur intestinal ; 2 : voie afférente représentée par le nerf vague ; 3 : centre de commande, représenté surtout par le noyau ventromédian de l'hypothalamus (VMH) : 4 et $4^{\prime}$ : voie afférente représentée par le nerf vague (rôle principal) et le nerf splanchnique.

départ en est constitué par les glucorécepteurs vagaux. Leur stimulation déclenche une augmentation rapide de la sécrétion d'insuline par l'intermédiaire des nerfs vagues (Mei et al., 1981) et des centres principaux comprenant l'aire latérale et surtout le noyau ventromédian de l'hypothalamus. Ce type de mécanismes, qui pourrait être très général dans le territoire digestif pré-absorptif, s'inscrit dans la catégorie des réflexes dits anticipateurs, étudiés surtout jusqu'ici dans la sphère céphalique (Nicolaidis, 1978) ; ils préparent et potentialisent les effets des méca- 
nismes plus tardifs, qui sont produits par l'arrivée des nutriments dans le territoire post-absorptif.

\section{4) La régulation du stockage des nutriments.}

A la lumière des recherches contemporaines conduites chez l'animal, le rôle du système nerveux doit être également envisagé dans le stockage des réserves glucidiques et lipidiques.

A) Les réserves glucidiques. - En l'absence de toute libération d'adrénaline, il a été bien démontré que la stimulation des nerfs vagues et celle des nerfs splanchniques retentissent sur le stockage du glycogène hépatique d'une façon diamétralement opposée. En effet, les nerfs vagues renforcent la glycogenèse alors que les nerfs splanchniques stimulent la glycogénolyse (Edwards, 1971). Jusqu'à ces dernières années, on pensait que ce mécanisme nerveux avait pour point de départ les glucorécepteurs hypothalamiques. Sans exclure la participation des récepteurs centraux, les travaux récents (Niijima, 1979) suggèrent l'implication des récepteurs périphériques: les glucorécepteurs hépatiques (Niijima, 1969) mais aussi les glucorécepteurs intestinaux (Niijima, 1985). II semble que les récepteurs polymodaux de l'intestin interviennent également, au début des repas, pour stimuler la glycogénolyse et favoriser ainsi le stockage ultérieur du glucose (Mei, résultats non publiés).

B) Les réserves lipidiques. La participation du système nerveux dans la gestion des réserves lipidiques a été envisagée au niveau du tissu adipeux brun. La richesse de ce tissu en fibres nerveuses sympathiques et en récepteurs adrénergiques a été soulignée par de nombreux auteurs (voir Seydoux et al., 1977). Le système sympathique jouerait un rôle capital, au moins chez certaines espèces, dans le contrôle du métabolisme du tissu adipeux brun, très largement impliqué dans la thermorégulation et la régulation pondérale (Jéquier, 1985). En effet, sa

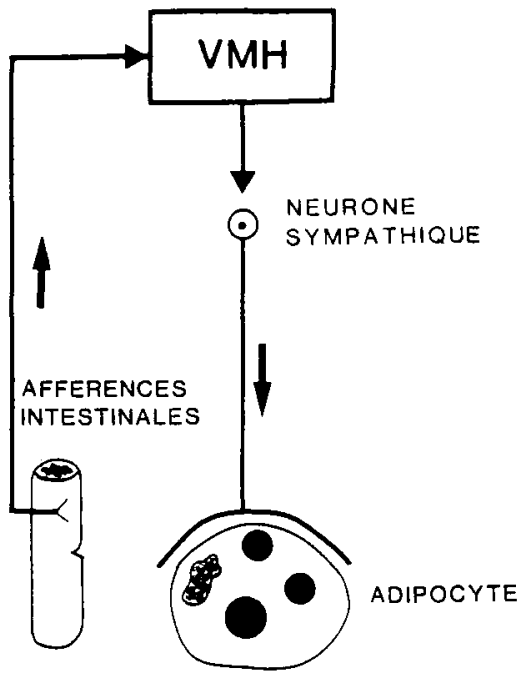

FIG. 2. - Contrôle nerveux du métabolisme du tissu adipeux brun. Les neurones sympathiques sont sous le contrôle du VMH qui reçoit lui-même des afférences de tout le territoire viscéral (afférences intestinales en particulier). 
stimulation électrique s'accompagne d'une activation de la lipolyse comme de la lipogenèse. Comme ces effets sont aussi obtenus par stimulation du noyau ventro-médian de I'hypothalamus (Takahashi et Shimazu, 1982), on peut se demander si les afférences digestives, qui projettent abondamment dans ce noyau, ne contribuent pas à cette régulation (fig. 2). Dans l'affirmative, les récepteurs aux lipides, récemment découverts dans l'intestin (Melone et al., 1985) constitueraient la source privilégiée de ce type d'informations et joueraient un rôle équivalent aux glucorécepteurs hépatiques dans le contrôle de la glycémie.

\section{Conclusion.}

Les données actuellement disponibles montrent que le système nerveux autonome est susceptible de contrôler le transit gastro-intestinal - ce qui était bien établi depuis longtemps - mais également la vasomotricité gastro-intestinale, les sécrétions exocrines et endocrines, et même l'absorption et le stockage des nutriments. Bien que ces données restent encore parcellaires, on peut donc affirmer que les systèmes nerveux extrinsèque et intrinsèque sont largement impliqués dans la nutrition.
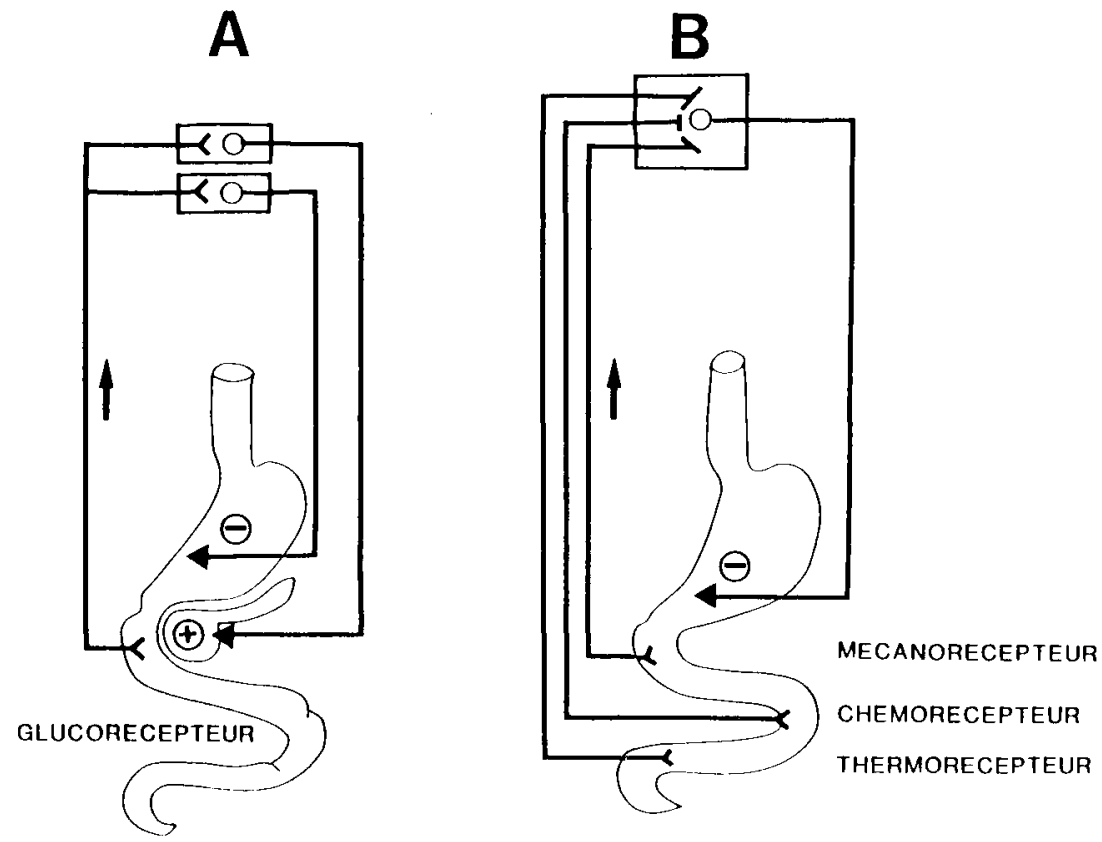

FIG. 3. - Implication des afférences intestinales dans les régulations digestives et homéostasiques. A : Un même type d'afférences, provenant des glucorécepteurs intestinaux interviennent dans 2 mécanismes différents : inhibition de l'activité gastrique $(-)$, activation de la sécrétion d'insuline $(+)$.

B : Plusieurs types d'afférences (issues respectivement des mécano - des chémo - et des thermorécepteurs intestinaux) participent ici à la même fonction : inhibition de l'activité gastrique. 
L'importance plus grande que l'on doit accorder au système nerveux, relativement aux autres mécanismes, apparaît plus conforme à l'ensemble de ses caractéristiques morpho-fonctionnelles. Elle s'explique notamment par la richesse remarquable de l'équipement sensoriel du tractus gastro-intestinal. Les nombreuses informations de toute nature qui y sont engendrées dans les conditions physiologiques constituent le point de départ de diverses régulations nerveuses. Deux modalités principales peuvent être distinguées suivant qu'une ou plusieurs sortes d'afférences sont impliquées.

Parfois, un même type d'afférences prend part à deux mécanismes différents. C'est le cas par exemple (fig. 3A) des afférences glucosensibles de l'intestin qui interviennent dans la régulation de la vidange gastrique et dans la régulation de la sécrétion d'insuline. D'autres fois, le phénomène inverse s'observe : des afférences de nature différente concourent au maintien d'une même fonction. L'exemple de la régulation nerveuse de l'évacuation gastrique est, à cet égard, très démonstratif (fig. 3B).

Ainsi, la richesse de la sensibilité digestive ne représente pas seulement une particularité anatomique : elle est aussi l'expression d'une importante participation fonctionnelle.

Les données expérimentales contemporaines qui ont abouti à cette conclusion, permettent de mieux comprendre le rôle du système nerveux dans la genèse des pathologies nutritionnelles (voir Rohner-Jeanrenaud et al., 1983). Finalement, elles vont dans le sens des connaissances actuelles montrant la contribution générale du système nerveux autonome au contrôle de la motricité des viscères, mais aussi, à la régulation de l'homéostasie et du comportement.

Journées Ingestion, Digestion, Absorption de I'Association française de Nutrition, Marseille, 5-6 décembre 1985.

\section{Références}

AGOSTONI E., CHINNOCK J. E., DALY M. de B., MURRAY J. G., 1957. Functionnal and histological studies on the vagus nerve and its branches to the heart, lungs and abdominal viscera in the cat. J. Physiol. (London), 135, 192-205.

CHOU C. C., BURNS T. D., HSIEH C. P., DABNEY J. M., 1972. Mechanisms of local vasodilatation with hypertonic glucose in the jejunum. Surgery, 71, 380-387.

EDWARDS A. V., 1971. The glycogenolytic response to stimulation of the splanchnic nerves in adrenalectomized calves, sheep, dogs, cats and pigs. J. Physiol. (London), 213, 741-759.

FURNESS J. B., COSTA M., 1974. The adrenergic innervation of the gastro-intestinal tract. Ergebn. Physiol., 69, 1-51.

FURNESS J. B., COSTA M., 1980. Types of nerves in the enteric nervous system. Neuroscience, 5, 1-20.

GERSHON M. D., 1981. The enteric nervous system. Am. Rev. Neurosci, 4, 227-272.

GONELLA J., 1978. La motricité digestive et sa régulation nerveuse. J. Physiol. (Paris), 74, 131-140.

GROSSMAN M. I., 1979. Neural and hormonal regulation of gastrointestinal function : an overview. Ann. Rev. Physiol., 41, 27-33.

HAJJAR J. J., SCHEDL H. P., 1981. Amino acid influx across the mucosal border of the rat intestine in vivo. Biochim. Biophys. Acta, 649, 759-768.

JÉQUIER E., 1985. Thermogenèse induite par les nutriments chez l'homme: son rôle dans la régulation pondérale. J. Physiol. (Paris), 80, 129-140. 
KELLY K. A., 1981. Motility of the stomach and gastroduodenal junction, 393-410. In JOHNSON L. R., Physiology of the gastrointestinal tract, Raven Press, New York.

LAPLACE J. P., CUBER J. C., 1984. Déafférentation vagale totale et évacuation gastrique chez le Porc. Reprod. Nutr. Dévelop., 27, 655-670.

LEEK B. F., 1977. Abdominal and pelvic visceral receptors. Br. Med. Bull., 33, 163-168.

MEI N., 1983. Sensory structures in the viscera. 1-42. In OTTOSON D., Progress in sensory physiology, Springer Verlag, Berlin.

MEI N., 1985. Intestinal chemosensitivity. Physiol. Rev., 65, 211-237.

MEI N., CONDAMIN M., BOYER A., 1971. The composition of the vagus nerve of the cat. Cell Tissue Res., 209, 423-431.

MEI N., ARCHAC A., BOYER A., 1981. Nervous regulation of insulin release by the intestinal vagal glucoreceptors. J. auton. nerv. Syst., 41, 351-363.

MELONE J., MEI N., GARNIER C., 1985. Données préliminaires sur l'existence de récepteurs intestinaux vagaux sensibles aux lipides. J. Physiol. (Paris), sous presse.

MORIN G., 1935. L'automatisme intestinal des Vertébrés et sa régulation. Th. Doct. ès-Sc., Lyon.

NICOLAIDIS S., 1978. Rôle des réflexes anticipateurs oro-végétatifs dans la régulation hydrominérale et énergétique. J. Physiol. (Paris), 74, 1-29.

NIIJIMA A., 1969. Afferent impulse decharges from glucoreceptors in the liver of the guinea pig. Ann. N. Y. Acad. Sci, 157, 690-700.

NIIJIMA A., 1979. Control of liver function and neuroendocrine regulation of blood glucose levels, 68-83. In Integrative functions of the autonomic nervous system. BROOKS C. MC, KOIZUMI K., SATO A., Elsevier, Amsterdam.

NIIJIMA A., 1985. Blood glucose levels modulate efferent activity in the vagal supply to the rat liver. J. Physiol. (London), 364, 105-112.

PAINTAL A. S., 1973. Vagal sensory receptors and their reflex effects. Physiol. Rev., 53, 159-227.

ROHNER-JEANRENAUD F., BOBIONI E., IONESCU E., SAUTER J. F., JEANRENAUD B., 1983. Central nervous system regulation of insulin secretion. Am. J. Physiol., 244, E317-322.

ROMAN C., GONELLA J., 1981. Extrinsic control of digestive tract motility, 289-333. In JOHNSON L. R., Physiology of the gastrointestinal tract, Raven Press, New York.

SEYDOUX J., CONSTANTINIDIS J., TSACOPOULOS M., GIRARDIER C., 1977. In vitro study of the control of the metabolic activity of brown adipose tissue by the sympathetic nervous system. J. Physiol. (Paris), 73, 985-996.

SINGER M. V., SOLOMON T. E., WOOD J., GROSSMAN M. I., 1980. Latency of pancreatic enzyme response to intraduodenal stimulants. Am. J. Physiol., 238, 23-29.

SJÖVALL H., REDFORS S., HALLBÄCK D. A., EKLUND S., JODAL M., LUNDGREN O., 1983a. The effect of splanchnic nerve stimulation on blood flow distribution, villous tissue osmolality and fluid and electrolyte transport in the small intestine of the cat. Acta Physiol. scand., 117, 359-365.

SJÖVALL H., REDFORS S., JODAL M., LÜNDGREN O., 1983b. On the mode of action of the sympathetic fibres on intestinal fluid transport: Evidence for the existence of a glucosestimulated secretory nervous pathway in the intestinal wall. Acta physiol. scand., 119, 39-48.

TAKAHASHI A., SHIMAZU T., 1982. Hypothalamic regulation of lipid metabolism in the rat: effect of hypothalamic stimulation on lipogenesis. J. auton. nerv. Syst., 6, 225-235.

TEPPERMAN B. L., JACOBSON E. D., 1981. Mesenteric circulation, 1417-1336. In JOHNSON L. R., Physiology of gastrointestinal tract, Raven Press, New York.

THOMAS J. E., 1957. Mechanics and regulation of gastric emptying. Physiol. Rev., 37, 453-474.

THOMAS E. M., TEMPLETON D., 1981. Noradrenergic innervation of the villi of rat jejunum. $J$. auton. nerv. Syst., 3, 25-29.

VATNER S. F., FRANKLIN D., VAN CITTERS R. L. 1970. Mesenteric vasoactivity associated with eating and digestion in the conscious dog. Am. J. Physiol, 219, 170-174.

WOOD J. D. 1981. Physiology of the enteric nervous system, 1-37, In JOHNSON L. R., Physiology of the gastrointestinal tract, Raven Press, New York.

YU Y. M., YU L. C. C., CHOU C. C. 1975. Distribution of blood flow in the intestine with hypertonic glucose in the lumen. Surgery, 178, 520-525. 